\title{
Silicon-on-Insulator (SOI) Ring Resonator-Based Integrated Optical Hydrogen Sensor
}

\author{
N. A. Yebo, Student Member, IEEE, D. Taillaert, Member, IEEE, J. Roels, Student Member, IEEE, D. Lahem, \\ M. Debliquy, D. Van Thourhout, Member, IEEE, and R. Baets, Fellow, IEEE
}

\begin{abstract}
We demonstrate a novel, highly sensitive integrated hydrogen sensor based on a silicon ring resonator on the silicon-oninsulator platform. The hydrogen sensitive element in the sensor structure is a platinum-doped tungsten oxide catalytic coating. The catalytic combustion of hydrogen in air leads to a local temperature rise in the ring resonator. The resulting thermo-optic effect shifts the ring resonator resonance. Resonance wavelength shifts higher than a nanometer are measured for hydrogen concentrations below the $4 \%$ lower explosion limit. A potentially tunable sensitivity of around $480 \mathrm{pm}$ shift per $\% \mathrm{H}_{2}$ is achieved at an operating temperature of about $40{ }^{\circ} \mathrm{C}$.
\end{abstract}

Index Terms-Integrated optics, lower explosion limit (LEL), resonance shift, thermo-optic, tungsten oxide $\left(\mathrm{WO}_{3}\right)$.

\section{INTRODUCTION}

$\mathbf{T}$ HE demand for clean energy is a major concern in today's environmentally aware world. Hydrogen gas has attracted a significant interest in this respect. Combustion of hydrogen in air produces heat energy with water as the only by-product. However, the very volatile and flammable nature of the gas has a negative impact on its safe implementation for public use as a fuel. A 4\% hydrogen concentration in air, which is sufficient to create explosions, is often termed as the lower explosion limit (LEL).

The majority of the optical hydrogen sensors have been based on optical fibers [1], [2]. Though these sensors interestingly provide most of the advantages of optical sensors, such as immunity from electromagnetic interference, multiplexing capabilities and good sensitivity, they are less suited for integration. The capability to integrate sensors, on the other hand, gives the opportunity to exploit more advantages such as, high compactness and inexpensive fabrication.

High index contrast (HIC) silicon-on-insulator (SOI) photonic devices have recently attracted considerable attention because of their ultrahigh compactness and compatibility to complementary metal-oxide-semiconductor (CMOS) fabrication technologies [3], [4]. HIC SOI ring resonators, in

Manuscript received November 04, 2008; revised March 01, 2009. First published April 21, 2009; current version published June 24, 2009. This work was supported in part by the European Union in the framework of the IST- ePIXnet Network of, Excellence and the Silicon Photonics Platform.

N. A. Yebo, D. Taillaert, J. Roels, D. Van Thourhout, and R. Baets are with the Department of Information Technology, Ghent University, 9000 Gent, Belgium (e-mail: nyebo@intec.ugent.be).

D. Lahem and M.Debliquy are with the Cellule Capteurs, Materia Nova, ASBL, Mons 7000, Belgium.

Color versions of one or more of the figures in this letter are available online at http://ieeexplore.ieee.org.

Digital Object Identifier 10.1109/LPT.2009.2021073 particular, are interesting for integrated sensing applications; they can be very small and sensitive [5]-[7]. In addition, they respond to the surrounding effects through a shift in the resonance wavelength, providing an inherent multiplexing feature for the implementation of sensor arrays.

Integrated optical hydrogen sensors reported to date use straight narrow waveguides as the basic sensing element [8]. Such sensors, however, are limited by the minimum length of wire set by the detection threshold. They are, in addition, less suited for multiplexing since they measure the change in the optical power rather than the shift in the operating wavelength.

In this letter, we demonstrate a novel, highly sensitive integrated hydrogen sensor based on an SOI ring resonator structure. In this scheme, platinum $(\mathrm{Pt})$-doped tungsten oxide $\left(\mathrm{WO}_{3}\right)$ coating is used as a hydrogen-sensitive element instead of the commonly used palladium in most hydrogen sensors; hysteresis and other limiting issues are often reported with sensors using palladium [1]. Furthermore, a good selectivity to hydrogen with respect to humidity and other combustible gases, such as $\mathrm{CO}$ and $\mathrm{CH}_{4}$, was recently reported for our coating [2]. To our knowledge, this is a first demonstration of a highly sensitive, integrated hydrogen sensor on SOI ring resonators.

\section{SENSOR BASICS}

In optical ring resonators, the guided modes respond with resonance wavelength shifts to the surrounding physical effects. From the condition for resonance, the corresponding shift in the resonance wavelength is given by

$$
\frac{\Delta \lambda}{\lambda}=\frac{\Delta L}{L}+\frac{\Delta n_{\mathrm{eff}}}{n_{\mathrm{eff}}}
$$

where $\lambda, n_{\text {eff }}$, and $L$ are the resonance wavelength, the effective mode index, and the round-trip length of the ring, respectively. Accordingly, either a change in effective index of the modes or a change in physical dimension of the ring can be exploited for sensing applications.

Fig. 1 schematically shows a scanning electron micrograph (SEM) of a microring resonator, and the cross-sectional view of the SOI hydrogen sensor structure in which the $\mathrm{WO}_{3}$ hydrogen sensitive layer is coated on a silica-clad SOI ring resonator.

Silicon ring resonators are typically very sensitive to temperature due to the high thermo-optic coefficient of silicon, around $1.8 \times 10^{-4} / \mathrm{K}$. We have taken advantage of this fact for sensing the presence of very low concentrations of hydrogen in air. Hydrogen gas is burned in air with the aid of Pt-doped $\mathrm{WO}_{3}$ catalytic coating on the $\mathrm{SiO}_{2}$ clad, silicon core ring resonator. The resulting heat is felt through a change in effective index of the 


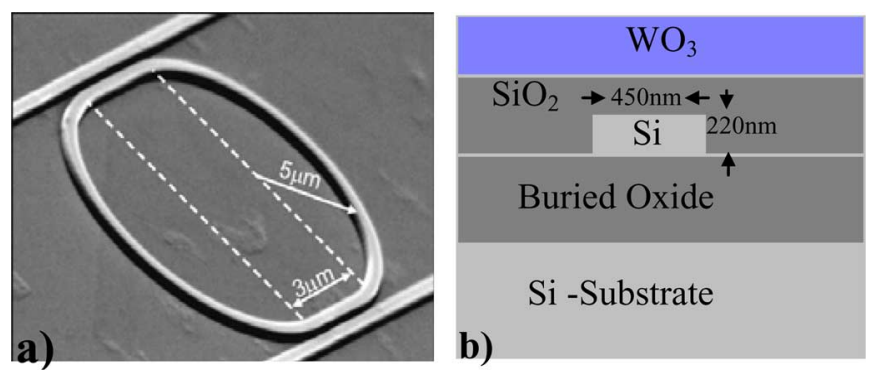

Fig. 1. (a) SEM picture of an air-clad microring resonator with $5-\mu \mathrm{m}$ bend radius. (b) Cross-sectional view of the SOI ring resonator-based hydrogen sensor.

guided mode leading to a shift in the resonance wavelength. The top $\mathrm{SiO}_{2}$ clad is used to avoid excess optical losses by preventing the direct interaction between the waveguide mode and the sensitive coating.

The chemical reaction involved at the top hydrogen sensitive $\mathrm{WO}_{3}$ layer is simply given by [2]

$$
2 \mathrm{H}_{2}+\mathrm{O}_{2} \leftrightarrow 2 \mathrm{H}_{2} \mathrm{O}+\text { Heat. }
$$

Heat transfer simulations in COMSOL multiphysics accompanied by optical simulations in FIMMWAVE have been done to predict the resulting effective index change for a given temperature rise on the top of the structure. An 80-pm/K resonance shift is estimated based on these simulations. This value is very close to the experimental result reported in an earlier work [3]. The simulations are run for a structure with 720-nm-thick top $\mathrm{SiO}_{2}$ clad and 220-nm-thick silicon core.

\section{FABRICATION}

The microring resonator structures are formed by patterning and etching a 220-nm-thick Si top layer on an SOI wafer using 193-nm deep ultraviolet optical lithography in a standard CMOS fabrication process [3], [4]. The waveguide structures are made to have the lateral cross section of $450 \mathrm{~nm}$ to achieve a single transverse-electric (TE)-mode operation. The buried $\mathrm{SiO}_{2}$ layer has a thickness of $2 \mu \mathrm{m}$. Grating couplers, which selectively couple TE modes from an optical fiber, are fabricated at the in-coupling and out-coupling sides of the bus waveguides. The use of grating couplers allows wafer/chip-scale testing of integrated optical circuits without cleaving or using external optics for coupling [5]. Also a relatively high alignment tolerance is achieved with these couplers.

The $\mathrm{WO}_{3}$ hydrogen sensitive material is prepared using the sol-gel method, and a layer of a few microns is dip coated on the top $\mathrm{SiO}_{2}$ cladding [2].

The ring resonators are designed to operate in the telecommunication wavelength range around $1550 \mathrm{~nm}$. The fabricated race-track ring resonators have a $5-\mu \mathrm{m}$ radius with a free spectral range of about $20 \mathrm{~nm}$. The measured $Q$-factor is over 25000 . Such a high $Q$-factor allows resolving very small resonance shifts down to $60 \mathrm{pm}$; hence, higher sensitivity is promised.

\section{EXPERIMENTS}

The sensor structure is placed inside a gas chamber which is sealed with a glass window. Input light from a Tunics Plus

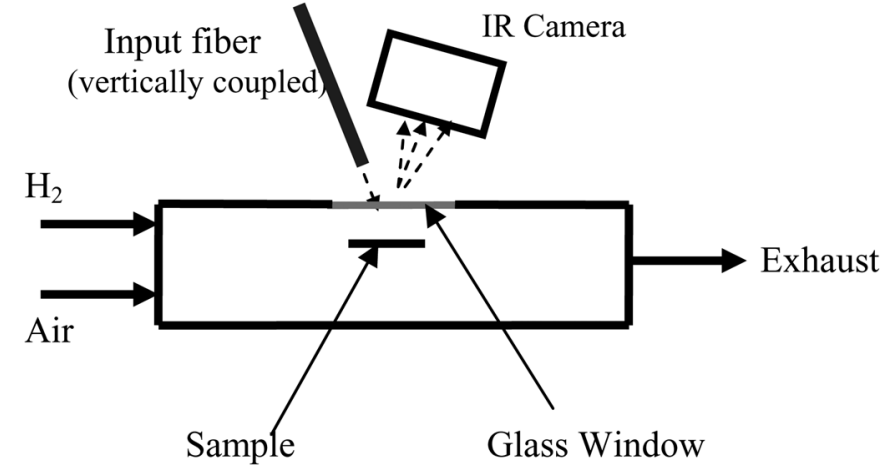

Fig. 2. Experimental setup.

tunable laser is vertically coupled through the glass window to the ring resonator. Coupling to the sensor structure from an optical fiber is achieved with a grating coupler [5]. The output light from the structure is collected by a XenIcs (Xeva-511) infrared (IR) camera via the output grating coupler. Measurement with an IR camera avoids the need for alignment to an output fiber through the glass window. It can also be used to read from several sensors on the same chip at a time.

The air and hydrogen gas flow into the chamber is controlled with two flow controllers. The exhaust valve on the other side of the chamber ensures continuous gas flow. The experimental setup is depicted in Fig. 2.

An air flow of constant rate of choice is maintained in the gas chamber containing the sample. Due to the fact that exothermic reactions are inherently dependent on the surrounding temperature and humidity, constant heating of the catalyst is required to facilitate the combustion of hydrogen in air.

Before the introduction of the hydrogen gas, the sample is heated by shining light from ordinary halogen lamp through the glass window. The resulting steady state sample temperature is estimated from the corresponding shift in the resonance wavelength of the ring. About $20^{\circ} \mathrm{C}$ rise above the room temperature is achieved from such heating. Finally, a hydrogen gas of a given concentration is introduced into the chamber and the resulting resonance shift is measured. Fig. 3 shows a measurement taken at different concentrations of hydrogen with an air flow kept at $1.3 \mathrm{~L} / \mathrm{min}$ and an approximate sample temperature of $40^{\circ} \mathrm{C}$

With such a configuration, significantly high sensitivity and stable sensor operation is achieved for hydrogen concentrations below the LEL. A fairly linear resonance shift of around $480 \mathrm{pm}$ per $\% \mathrm{H}_{2}$ within an accuracy of $\pm 60 \mathrm{pm}$ is achieved. More noticeably, a $1.2-\mathrm{nm}$ resonance shift is measured for $3 \%$ hydrogen in air. A detection threshold of $0.7 \% \mathrm{H}_{2}$ is estimated from the sensitivity curve in Fig. 3(b) and the $Q$ factor of the ring resonator.

Noting the enhancement of the catalytic activity of the coating with temperature, one can expect to lower the detection threshold by operating at relatively higher sample temperatures [9].The detailed sensitivity enhancement with temperature is, however, further to be investigated experimentally. Heating with the halogen lamp has also demonstrated the opportunity for optical means of maintaining constant sample temperature. Integrated gratings which continuously leak light to the sensi- 

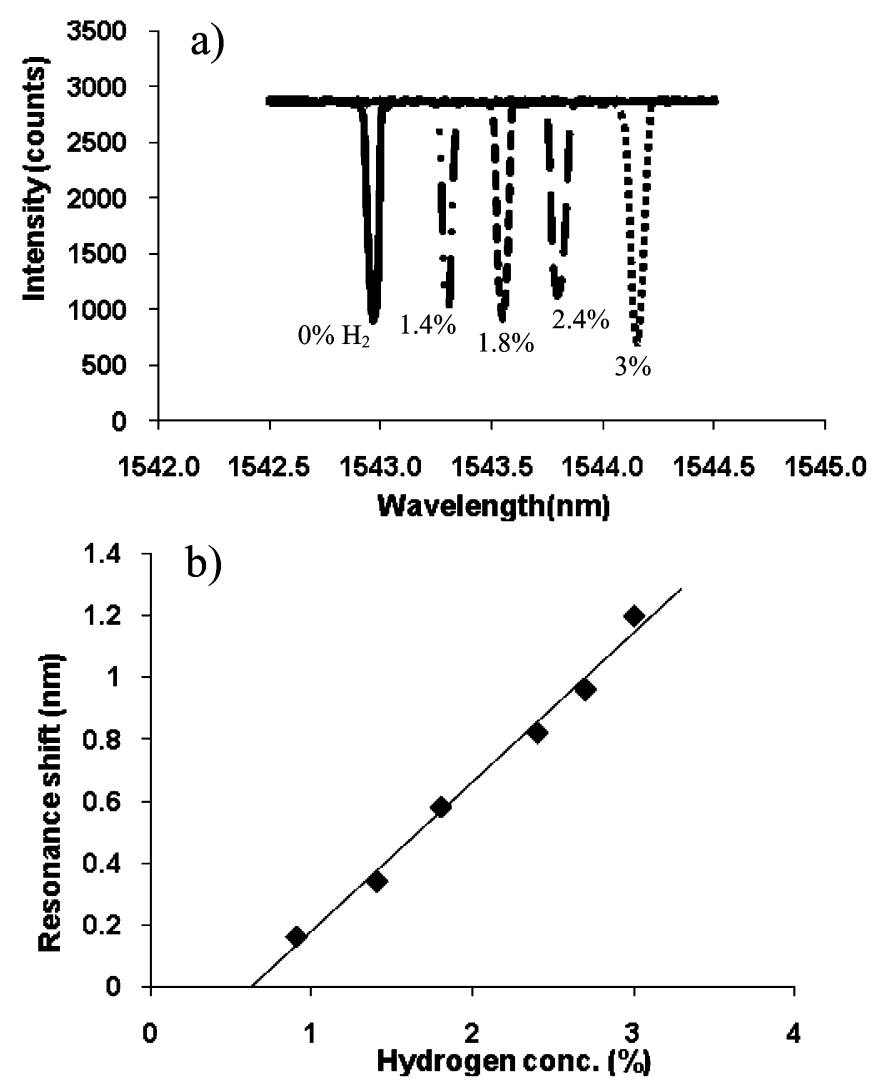

Fig. 3. (a) Measured spectrum of the ring resonance. (b) Measured ring resonance shifts, at different hydrogen concentrations. The solid line in (b) represents the linear fit of the measured data.

tive coating might also be considered to achieve the required heating effect in a compact way.

Approximately one minute is needed for each measurement due to the time required to tune the laser over a measurement window of interest. However, based on the recent result by the coworkers for the same coating on fiber Bragg gratings, we estimate a subsecond scale or a bit higher response to be achievable on a relatively faster measurement setup [2]. The microsecond scale heat transfer from the top layer to the core, simulated in COMSOL, predicts an even faster intrinsic response of the SOI ring resonator. The excess response time can be associated with the measurement units and the coating.
For a more convenient and faster read-out system, the sensor can be fiber coupled to a broadband source and an external spectrometer, or an integrated spectrometer can be used [10]. A second ring resonator without the sensitive coating can also be fabricated in series with the sensor as a reference to the wavelength drifts due to the surrounding temperature.

With ongoing progress to integrate optical sources, filters, and detectors directly on a chip, the inexpensive mass fabrication of our sensor might be possible in the future.

\section{CONCLUSION}

An integrated hydrogen sensor was demonstrated using an SOI ring resonator. Resonance wavelength shifts over a nanometer are achieved for hydrogen concentrations below the LEL. High sensitivity, compactness, tunable sensitivity, and the promise for inexpensive fabrication are the attractive features about the hydrogen sensor.

\section{REFERENCES}

[1] M. Tabib-Azar, "Highly sensitive hydrogen sensors using palladium coated fiber optics with exposed cores and evanescent field interactions," Sens. Actuators B, Chem., vol. 56, pp. 158-163, Jun. 1999.

[2] C. Caucheteur, "Catalytic fiber Bragg grating sensor for hydrogen leak detection in air," IEEE Photon. Technol. Lett., vol. 20, no. 2, pp. 96-98, Jan. 15, 2008.

[3] P. Dumon, "Linear and nonlinear nanophotonic devices based on silicon -on -insulator wire waveguides," Jpn. J. Appl. Phys., vol. 45, pp. 6589-6602, Aug. 2006.

[4] W. Bogaerts, "Nanophotonic waveguides in silicon-on-insulator fabricated with CMOS technology," J. Lightw. Technol., vol. 23, no. 1, pp. 401-412, Jan. 2005.

[5] G. Roelkens, "High efficiency diffractive grating couplers for interfacing a single mode optical fibre with silicon-on- insulator waveguide circuit," Appl. Phys. Lett., vol. 92, p. 131101, Apr. 2008.

[6] V. M. N. Passoro, "Ammonia optical sensing by microring resonators," Sensors, vol. 7, pp. 2741-2749, Nov. 2007.

[7] J. T. Robinson, "On-chip gas detection in silicon optical microcavities," Opt. Express, vol. 16, pp. 4296-4301, Mar. 2008.

[8] P. Tobiska, "An integrated optic hydrogen sensor based on SPR on palladium," Sens. Actuators B, Chem., vol. 74, pp. 168-172, Apr. 2001.

[9] V. K. Yatsimirskii, "Effect of Pt, Pd and $\mathrm{Cs}^{+}$on the surface state and catalytic activity of $\mathrm{Wo}_{3}$ in oxidation of hydrogen," Theor. Experimental Chem., vol. 41, pp. 135-138, Feb. 2005.

[10] J. Brouckaert, "Miniature integrated spectrometer fabricated on a silicon-on-Insulator substrate," in Proc. 21st LEOS Annual Meeting, Acapulco, 2008, pp. 55-56. 